\title{
A hygrothermo-mechanical model for wood: part A. Poroelastic formulation and validation with neutron imaging
}

\section{COST Action FP0904 2010-2014: Thermo-hydro-mechanical wood behavior and processing}

\begin{abstract}
The correct prediction of the behavior of wood components undergoing environmental loading or industrial process requires that the hygrothermal and mechanical (HTM) behavior of wood is considered in a coupled manner. A fully coupled poromechanical approach is proposed and validated with neutron imaging measurements of moist wood specimens exposed to high temperature. This paper demonstrates that a coupled HTM approach adequately captures the variations of temperature, moisture content, and dimensions that result in a moist wood sample exposed to one-side heating.
\end{abstract}

Keywords: energy method, finite element method (FEM), heat and mass transport, hygrothermal and mechanical (HTM) behavior, neutron imaging, poromechanics

DOI 10.1515/hf-2014-0189

Received June 30, 2014; accepted March 12, 2015; previously published online April 17, 2015

\section{Introduction}

An essential characteristic of wood as a porous material of biological origin is its interaction with moisture. In the

*Corresponding author: Dominique Derome, Laboratory of Multiscale Studies in Building Physics, Empa, Uberlandstrasse 129, 8600 Dübendorf, Switzerland, e-mail: dominique.derome@empa.ch Saeed Abbasion: Department of Civil Engineering, ETHZ, 8093 Zürich, Switzerland

Jan Carmeliet: Chair of Building Physics, ETHZ, 8092 Zürich, Switzerland; and Laboratory of Multiscale Studies in Building Physics, Empa, 8600 Dübendorf, Switzerland

Marjan Sedighi Gilani: Applied Wood Materials, Empa, 8600 Dübendorf, Switzerland

Peter Vontobel: Spallation Neutron Source Division, Paul Scherrer Institute (PSI), 5232 Villigen, Switzerland hygroscopic domain, wood may sorb different moisture contents $(u)$ depending on the relative humidity $(\mathrm{RH})$ of the environment. The thermal gradients resulting from exposing wood to heat lead to moisture transport. The transport and storage of moisture and heat in wood are interrelated to each other (i.e., dependent on both temperature and $u$ ). Furthermore, changes in $u$ result in swelling/ shrinkage and softening/hardening of wood. Temperature changes have similar dimensional and mechanical effects, which are less pronounced.

One example for the hygrothermal and mechanical (HTM) loading of wood is friction welding. Here, two wood pieces are submitted to a relative motion to each other parallel to the welded plane for a few seconds; the joint interface heats up due to the friction, while, in a small layer, plastic deformation and partial chemical degradation occur. After cooling down, the two pieces are fixed to each other. The glassy, natural bonding interface formed of modified lignin and hemicelluloses is strengthened by entangled wood fibers. In the present paper, a mathematical modeling of the heat, moisture, and mechanical aspects of the welding process is in focus.

The literature describes the modeling of friction welding for several materials, such metals, ceramics, and polymers. Usually, modeling focuses on the thermal effects and not on the mechanical effects. However, Sluzalec (1990) considered both effects, thus computing strain and stress fields and temperature $(T)$ distribution in welded pieces. Fu and Duan (1998) applied the finite element method (FEM) to analyze coupled deformation and heat flow. D'Alvise et al. (2002) included contact algorithm and automatic re-meshing at the joint interface and considered a formulation for the friction law including thermo-dependent material consistency. Lindemann et al. (2006) modeled the bonding of aluminum with corundum ceramic. For modeling wood-dowel rotation welding, an analytical approach was applied (Zoulalian and Pizzi 
2007), where the heat source term contains a constant friction coefficient. Ganne-Chédeville et al. (2008) developed a numerical FEM to simulate the $T$ behavior of wood pieces during linear welding. The heat flow at the interface was imposed to produce the $T$ measured with infrared thermography (IRT) at the side surfaces of two wood pieces during welding. This model did not include mechanical aspects.

The full welding process has been seldom analyzed by FEM, but studies more and more consider both HTM aspects of porous materials during welding. In the field of cementitious materials, using the hybrid mixture theory, Gawin et al. (2003) analyzed the hygrothermal behavior at high $T$ accounting for mechanical material deterioration. Zeng et al. (2011) developed a poromechanical approach of freezing behavior. The comprehensive model of Rémond et al. (2007) links heat and mass transport to the mechanical behavior of wood, but the model is not fully coupled.

The welding of wood is very complex, and the aim of this paper is to propose a model that captures, in a first step, the physical aspects involved in wood welding, disregarding actual friction, and any chemical transformation. The poromechanical approach will be applied to consider simultaneously the hygric, thermal, and mechanical (i.e., HTM) behavior. The transport of heat and mass will be described using $T$ and capillary pressure gradients as the driving forces. Then, a heating experiment will be documented based on neutron imaging (NI), which was specially designed to allow a validation of the proposed model. The subsequent paper of this series is dealing with the application of the model in parametric studies (Abbasion et al. 2015).

\section{Computational model}

The continuum approach of this study considers the macroscopic scale, where the porous wood appears to be homogeneous (i.e., where neither the different material components nor the cellular structure can be distinguished). However, in view of the growth ring structure of wood, a mesoscale feature is introduced to consider latewood (LW) and earlywood (EW). The orthotropic nature of wood is also taken into account. In this continuum approach, the porous material is considered to consist of three superimposed phases: (1) the solid phase (subscript $s$; i.e., the solid matrix), (2) the liquid phase (subscript $l$; i.e., water), and (3) the gaseous phase (subscript $g$; i.e., moist air), which is a perfect mixture of two ideal gasses: dry air (subscript $a$ ) and water vapor (subscript $v$ ).

\section{Poromechanical approach}

The poromechanics theory was introduced by Biot (1941) to take into account the interaction of fluids with the solid matrix. Coussy (1989,
1991, 1995, 2004, 2007, 2010) and Coussy et al. (1998) presented a general framework to formulate adequate constitutive equations for the poroelastic behavior. Here, a linear thermoporoelastic model is described first for unsaturated isotropic porous solids. The material is assumed to consist of a solid material (matrix) and a pore space partly filled by liquid water and a gas phase consisting of water vapor and dry air. The so-called Lagrangian porosity $\phi$ (Coussy 2007, 2010) relates the current porous volume of the material to the initial porous volume occupied before its deformation. The porosity is subdivided in the part filled by the liquid $\phi_{l}$ and the gas $\phi_{g}$. The moisture content $(u)$ is defined as the ratio of liquid to dry material masses, or $u=\rho_{l} \phi_{l} / \rho_{o}$, with $\rho_{l}$ and $\rho_{o}$ representing the density of the liquid and the dry material, respectively. The pressures in the liquid and gas phases are $p_{l}$ and $p_{g}$, respectively. The equivalent pressure, which is acting on the solid matrix, is denoted by $p$ and can be obtained by incremental volumetric averaging of the liquid and gaseous phases together, that is, $\phi \mathrm{d} p=\phi_{l} \mathrm{~d} p_{l}+\phi_{g} \mathrm{~d} p_{\mathrm{g}}$ or, in more common form, $\mathrm{d} p=\mathrm{d} p_{g}+S_{l} \mathrm{~d} p_{c}$, where $S_{l}=\phi_{l} / \phi$ is called liquid degree of saturation and $p_{c}=p_{g}-p_{l}$ is the capillary pressure. The material is assumed to remain elastic and is initially stress free at an initial temperature $T_{0}$ and pressure $p_{0}$. Following Coussy (2007), the free energy balance reads

$$
\sigma_{i j} \mathrm{~d} \varepsilon_{\mathrm{ij}}+p \mathrm{~d} \phi-s \mathrm{~d} T-\mathrm{d} F=0
$$

The first term refers to the elastic strain work related to the deformation of an infinitesimal element, with the $\sigma_{i j}$ (solid stress) and $\varepsilon_{i j}$ (solid strain) components. The second term accounts for the work supplied to the porous solid through its internal walls related to the change of porosity $\phi$. The third term is related to energy change due to $T$ change, with $s$ being the entropy. $F$ is the free energy of the solid matrix forming the solid part of the porous solid. Introducing the Legendre transform $W$ of $F$

$$
W(\sigma, p, T)=F(\varepsilon, \phi, T)-\sigma_{i j} \varepsilon_{i j}-p \phi
$$

Equation (1) becomes

$$
\varepsilon_{\mathrm{ij}} \mathrm{d} \sigma_{i j}+\phi \mathrm{d} p+s \mathrm{~d} T+\mathrm{d} W=0
$$

From Equation (3) the following is derived:

$$
\varepsilon_{i j}=-\frac{\partial W}{\partial \sigma_{i j}} ; \phi=-\frac{\partial W}{\partial p} ; s=-\frac{\partial W}{\partial T}
$$

Owing to the additive character of the energy $W$, it can be written as

$$
W=-\frac{1}{2} S_{i j k l} \sigma_{i j} \sigma_{k l}-\frac{1}{2} M \pi^{2}-C(\theta-\theta \ln \theta)-B_{i j} \sigma_{i j} \pi-\alpha_{i j} \sigma_{i j} \theta-\alpha_{\phi} \pi \theta
$$

The first term at the right-hand side of Equation (5) describes the elastic energy contribution, the second term the energy contribution of the fluid phases, and the third the energy change due to a change in $T$ related to the heat capacity. The fourth term describes the energy contribution due to the fluid-solid interaction, and the fifth and sixth terms describe the elastic energy contribution due to a thermal dilatation of the skeleton and the pore space, respectively. For linear poroelastic solids, the mechanical, thermal, and fluid terms read as in Equation (5), with $\pi=p-p_{0}$ and $\theta=T-T_{0}$, where $S_{i j k l}$ is the tensor of elastic compliances, $M$ is the moisture capacity, $C$ is the volumetric heat capacity, $\alpha_{i j}$ is the tensor of thermal dilatation coefficients, $B_{i j}$ is the tensor of moisture-mechanical coupling coefficients, and $\alpha_{\varphi}$ is the volumetric thermal dilatation related to the porosity. 
Substituting Equation (5) in Equation (4), the state equations for linear poroelasticity are derived:

$$
\begin{gathered}
\varepsilon_{i j}-\varepsilon_{i j}^{0}=S_{i j k l} \sigma_{k l}+B_{i j} \pi+\alpha_{i j} \theta ; \quad \phi-\phi^{0}=B_{i j} \sigma_{i j}+M \pi+\alpha_{\phi} \theta \\
s-s^{0}=\alpha_{i j} \sigma_{i j}+\alpha_{\phi} \pi+\frac{C}{T_{0}} \theta
\end{gathered}
$$

For isotropic materials, swelling and thermal expansion are assumed to be only volumetric, which means $B_{i j}=0$, and $\alpha_{i j}=0$, when $i \neq j$. Equation (6) can then be written in terms of the mean stress $\sigma$, mean volumetric strain $\varepsilon$, deviatoric stress $s_{i j}$, and deviatoric strain $e_{i j}$ :

$$
\varepsilon-\varepsilon^{0}=S \sigma+B \pi+3 \alpha \theta ; \quad \phi-\phi^{0}=B \sigma+M \pi+\alpha_{\phi} \theta ; \quad e_{i j}-e_{i j}^{0}=\frac{1}{2} D s_{i j}
$$

where $S$ is the bulk compliance, $B$ is the moisture-mechanical coupling coefficient, $M$ is the moisture capacity, and $D$ is the shear compliance. Equation (7) can be rewritten in the more classical poroelastic form as presented by Coussy (2004):

$$
\sigma-\sigma^{0}=K \varepsilon-3 \alpha K \theta-b \pi ; \quad \phi-\phi^{0}=b \varepsilon+\frac{p}{N}+\alpha_{\phi} \theta ; \quad s_{i j}-s_{i j}^{0}=2 G e_{i j}
$$

where $K$ is the bulk modulus, $b$ is the Biot coefficient, $N$ is the Biot modulus, and $G$ is the shear modulus.

A nonlinear poroelastic model for unsaturated orthotropic porous solids is described starting with the constitutive equations. The solid is now assumed to be nonlinear elastic and orthotropic, as done in Carmeliet et al. (2013), and extending to include the $T$ field. Using Equation (4) and with regard to symmetry of the second derivative of $W$, the incremental state equations for strain, moisture content, and entropy become

$$
\begin{gathered}
\mathrm{d} \varepsilon_{i j}=-\frac{\partial^{2} W}{\partial \sigma_{i j} \partial \sigma_{k l}} \mathrm{~d} \sigma_{k l}-\frac{\partial^{2} W}{\partial \sigma_{i j} \partial p} \mathrm{~d} p-\frac{\partial^{2} W}{\partial \sigma_{i j} \partial T} \mathrm{~d} T \\
\mathrm{~d} \phi=-\frac{\partial^{2} W}{\partial \sigma_{i j} \partial p} \mathrm{~d} \sigma_{i j}-\frac{\partial^{2} W}{\partial p^{2}} \mathrm{~d} p-\frac{\partial^{2} W}{\partial T \partial p} \mathrm{~d} T \\
\mathrm{~d} s=-\frac{\partial^{2} W}{\partial \sigma_{i j} \partial T} \mathrm{~d} \sigma_{i j}-\frac{\partial^{2} W}{\partial T \partial p} \mathrm{~d} p-\frac{\partial^{2} W}{\partial T^{2}} \mathrm{~d} T
\end{gathered}
$$

Defining the thermoporoelastic properties

$$
\begin{aligned}
& S_{i j k l}=-\frac{\partial^{2} W}{\partial \sigma_{i j} \partial \sigma_{k l}} \bar{B}_{i j}=-\frac{\partial^{2} W}{\partial \sigma_{i j} \partial p} \alpha_{k l}=-\frac{\partial^{2} W}{\partial \sigma_{i j} \partial T} \\
& M=-\frac{\partial^{2} W}{\partial p^{2}} \alpha_{\phi}=-\frac{\partial^{2} W}{\partial T \partial p} C=-T \frac{\partial^{2} W}{\partial T^{2}}
\end{aligned}
$$

the constitutive equations of the nonlinear porous solid are written as

$$
\begin{gathered}
\mathrm{d} \varepsilon_{i j}=S_{i j k l} \mathrm{~d} \sigma_{k l}+B_{i j} \mathrm{~d} p+\alpha_{i j} \mathrm{~d} T \\
\mathrm{~d} \phi=B_{i j} \mathrm{~d} \sigma_{i j}+M \mathrm{~d} p+\alpha_{\phi} \mathrm{d} T \\
\mathrm{~d} s=\alpha_{i j} \mathrm{~d} \sigma_{i j}+\alpha_{\phi} \mathrm{d} p+C \mathrm{C} T / T
\end{gathered}
$$

For the nonlinear material, the thermo-poroelastic properties $S_{i j k l}\left(\sigma_{i j}, p, T\right), B_{i j}\left(\sigma_{i j}, p, T\right), \alpha_{i j}\left(\sigma_{i j}, p, T\right), M\left(\sigma_{i j} p, T\right), \alpha_{\varphi}\left(\sigma_{i j}, p, T\right)$, and $C\left(\sigma_{i j}, p, T\right)$ generally depend on stress, $T$, and effective pressure.

Wood has two different pore systems: a fine nanosized one in the cell wall and a microscale pore network formed of the tracheid and ray lumens, which are connected by pits. In the cell wall at dry conditions, there is an initial porosity. Most of the porosity is, however, created during the adsorption of water molecules and the swelling of the cell wall. In the following, the cell wall is assumed to be always in saturated condition or $S_{l}=1$.
The constitutive behavior as described by Equations (11a) and (11b) under well-defined experimental conditions is now analyzed. Consider a first experiment, where the material is brought in an environment with a certain gas (air) pressure $p_{g}$, water vapor pressure $p_{v}$, and temperature $T$. The $\mathrm{RH}$ of the environment is defined as $\mathrm{RH}=p_{v} / p_{v s a t}(T)$, where $p_{v s a t}$ is the saturation vapor pressure related to $T$. Assuming equilibrium between the external vapor pressure and the vapor pressure in the pore space, Kelvin's law describes the relation between the pore vapor pressure (or RH) and the capillary pressure in the porous material:

$$
R H=\exp \left(p_{c} / \rho_{l} R_{v} T\right)
$$

where $R_{v}$ is the ideal gas constant for vapor. As mentioned above, the cell wall is assumed to be saturated (Maloney and Paulapuro 1999, i.e., $S_{1}=1$ ), and as experiments are performed at constant gas pressure (i.e., $\mathrm{d} p_{g}=0$ ), the increments in effective pressure are equal to capillary pressure increments. Assuming a constant external vapor pressure and the material in immediate equilibrium, the effective pressure inside the material remains constant or $\mathrm{d} p=0$. A mechanical load is then applied and the strain and change of $u$ are measured. Immediate equilibrium between the vapor pressure of the environment and the pore space of the material is assumed. Equation (11a) then gives $\mathrm{d} \varepsilon_{i j}=S_{i j k l} \mathrm{~d} \sigma_{k l}$ and $S_{i j k l}$ defines the elastic compliance, a measure for the elastic strain capacity of the material. When the mechanical test is performed at different effective (or capillary) pressures and $T \mathrm{~s}$, the compliance $S_{i j k l}\left(\sigma_{k l} p, T\right)$ dependent on stress, pressure, and $T$ can be determined. The $u$ increment is given by $\mathrm{d} u=\left(\rho_{l} / \rho_{0}\right) \mathrm{d} \phi$. For constant pressure and $T$ test conditions, Equation (11b) reads $\mathrm{d} u=\left(\rho_{l} / \rho_{0}\right) B_{i j} \mathrm{~d} \sigma_{i j}$. The coupling coefficient $B_{i j}$ describes the change in $u$ due to an external mechanical stress. The influence of mechanical stress on the sorption process is called the mechanosorptive effect.

In a second experiment, the stress and pressure are considered constant (neglecting time-dependent behaviors such as creep) and the material is exposed to environments with different $T$. The strain and change of $u$ are measured. Immediate equilibrium between the $T$ of the environment and the material is assumed. Equation (11a) then reads $\mathrm{d} \varepsilon_{i j}=\alpha_{i j} \mathrm{~d} T$ and describes the thermal dilatation. Equation (11b) reads $\mathrm{d} u=\left(\rho_{l} / \rho_{0}\right) \alpha_{\varphi} \mathrm{d} T$ and describes the change in $u$ due to $T$ change, which defines the volumetric thermal dilatation related to the porosity.

In a third experiment, the stress and $T$ are considered constant (once more neglecting time-dependent behaviors such as creep) and the material is exposed to environments with different external vapor pressure. The strain and change of $u$ are measured. Immediate equilibrium between the vapor pressure of the environment and the pore space of the material is assumed. Equation (11a) then reads $\mathrm{d} \varepsilon_{i j}=B_{i j} \mathrm{~d} p$ and describes the change in strain due to a change in liquid pressure. The coupling coefficient $B_{i j}$ thus describes the swelling/shrinkage of the material. Equation (11b) reads $\mathrm{d} u=\left(\rho_{l} / \rho_{0}\right) M \mathrm{~d} p . M$ describes the change in $u$ due to a change in effective (or capillary) pressure and is called the moisture capacity.

In a standard sorption test, the material is free of external load or $\sigma_{i j}=0$. The $u$ measured in the unloaded condition is referred to as $u_{0}$, where the subscript 0 refers to the zero stress condition. The moisture capacity normally measured in unloaded condition is given by

$$
\left(\rho_{l} / \rho_{0}\right) M_{0}=\partial u_{0} / \partial p
$$

Also, for the nonlinear elastic material, the energy $W$ may be described by the sum of six energy terms as given in Equation (5). For the linear elastic material, this energy is of quadratic form. However, 
for the nonlinear elastic material, the energy consists not only of quadratic terms but also of higher-order terms. According to Carmeliet et al. (2013), a general poroelastic description at constant $T$ for orthotropic materials is given for wood. The compliance is found to be a quadratic function of the $u$ or

$$
S_{i j}=S_{i j}^{0}+B_{i j}^{1} u_{0}^{2}
$$

$B_{i j}^{1}$ terms refer to the first-order coupling between stress and $u$ in the energy equation of Carmeliet et al. (2013). The values for $S_{i j}$ are normalized to the compliance in the longitudinal (L) direction $S_{11}^{0}$. $B_{i j}^{1}$ is normalized to the corresponding compliance $S_{i j}^{0}$ to yield

$$
S_{i j}=S_{11}^{0} a_{i j}\left(1+b_{i j} u_{0}^{2}\right)
$$

with the dimensionless parameters $a_{i j}$ and $b_{i j}$ given by

$$
a_{i j}=\frac{S_{i j}^{0}}{S_{11}^{0}}, b_{i j}=\frac{B_{i j}^{1}}{S_{i j}^{0}}
$$

The swelling strain is assumed to be only volumetric, so $B_{44}=B_{55}=B_{66}=0$. This assumption is correct for isotropic materials but is not proven for orthotropic materials. However, swelling in shear may be assumed negligible. The free swelling at stress-free conditions is given by $\mathrm{d} \varepsilon_{i i}=B_{i i}^{0} \mathrm{~d} u_{0}$, and the parameter $B_{i i}^{0}$ can be determined as the slope of the swelling strain versus $u$. Note that $B_{i j}^{0}$ describes the second-order coupling between stress and $u$ (Carmeliet et al. 2013).

Figure 1 compares measurements and curves obtained from the poroelastic model. An overall good agreement between measurement data and the poroelastic model is observed. Table 1 summarizes the values determined for $S_{11}^{0}, a_{i j}$, and $b_{i j}$. The coefficient $a_{i j}$ spans a wide range from -0.29 to 222 , showing the important orthotropic behavior of wood. The coefficient $b_{i j}$ describes the moisture sensitivity of the compliance. The compliance is most moisture sensitive in the tangential $(\mathrm{T})$ and radial (R) directions and much less in the L direction. The shear compliances are moisture sensitive, with a maximum in the RT direction. The off-diagonal compliances are very moisture sensitive. The swelling coefficient $B_{i i}^{0}$ is highest in the T direction, in the $\mathrm{R}$ direction half this value, and in the $\mathrm{L}$ direction very low. The orthotropic moisture-dependent behavior of wood may be explained by its composition. The geometry of the individual cells and the arrangement/structure of the cells across the growth ring are responsible in large part for the anisotropy of swelling and stiffness, the architecture of the cell wall layers and of the stiffer microfibril aggregates being the other factors affecting this anisotropy. Furthermore, at the mesoscale, the interaction of LW and EW may influence transverse anisotropy, as LW shows isotropic swelling, whereas EW swelling is greater in the $\mathrm{T}$ direction than in the R direction (Derome et al. 2011; Patera et al. 2013).

\section{Heat and mass transport}

For the conservation of mass in a porous material, the general mass balance equation for the mass component $w_{i}\left(\mathrm{~kg} \mathrm{~m}^{-3}\right)$ can be written as

$$
\frac{\partial w_{j}}{\partial t}=-\nabla \cdot \mathbf{g}_{j}+G_{i \rightarrow j}
$$
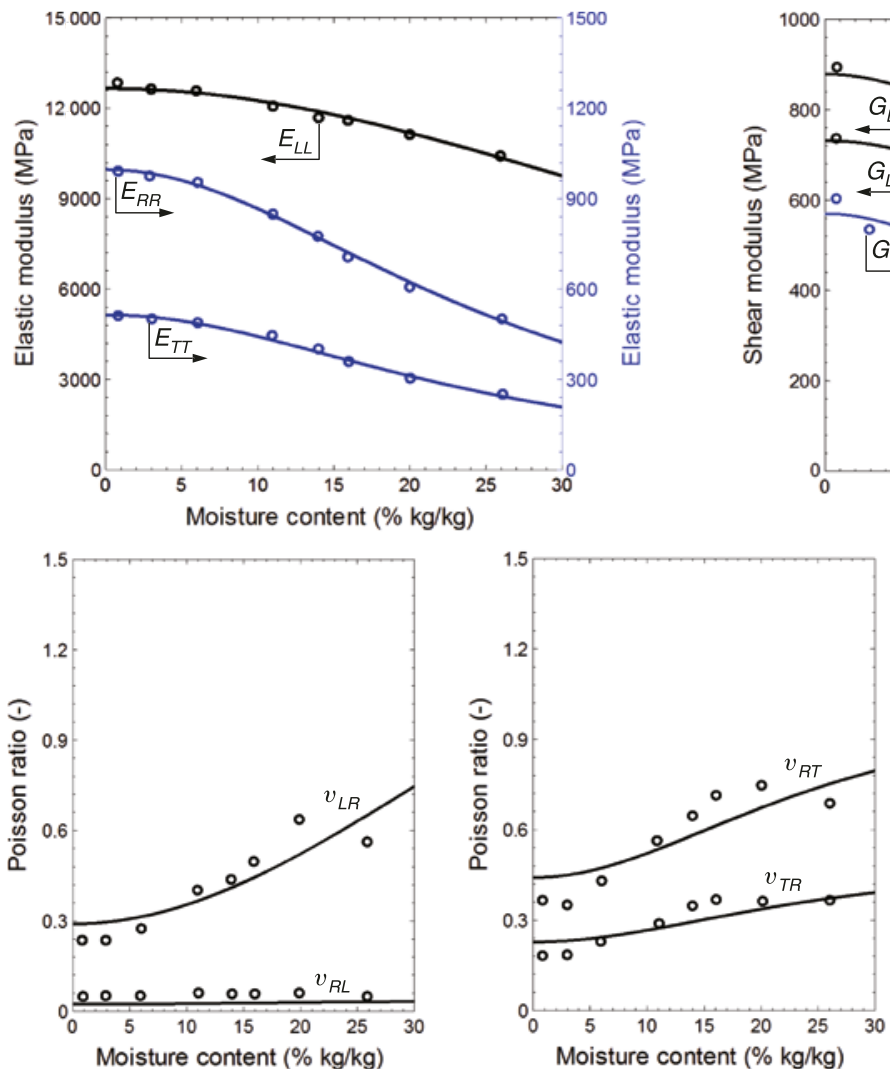
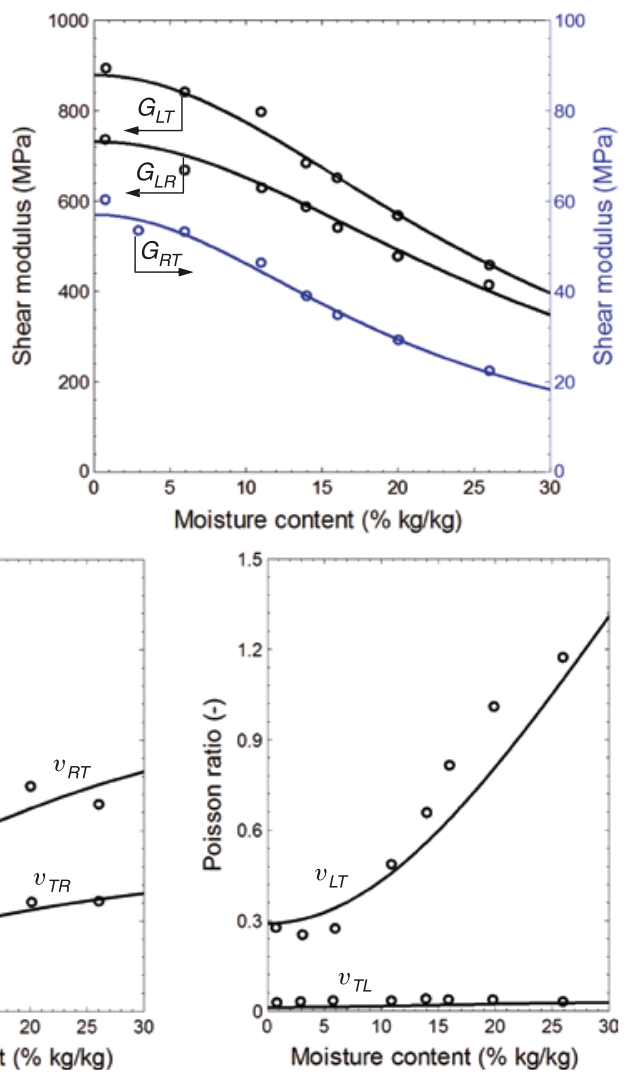

Figure 1: Poromechanical model results (continuous lines) fitted with experimental modulus of elasticity (circles) reported by Neuhaus (1981). 
Table 1: Poromechanical parameters of wood, $S_{11}^{0}=1 /(12660 \mathrm{MPa})$.

\begin{tabular}{rrrr}
\hline $\boldsymbol{S}_{i j}^{0}$ & $\boldsymbol{a}_{i j}$ & $\boldsymbol{b}_{i j}$ & $\boldsymbol{B}_{i i}^{0}$ \\
\hline $11(\mathrm{~L})$ & 1 & 3.3 & 0.03 \\
$22(\mathrm{R})$ & 12.7 & 15 & 0.14 \\
$33(\mathrm{~T})$ & 24.6 & 16.3 & 0.31 \\
$12(\mathrm{LR})$ & -0.29 & 26 & - \\
$13(\mathrm{LT})$ & -0.29 & 64 & - \\
$23(\mathrm{RT})$ & -5.6 & 36 & - \\
$4(\mathrm{RT})$ & 222 & 23.5 & 0 \\
\hline
\end{tabular}

where $t$ is the time (s), $\mathbf{g}_{j}$ is the flux vector $\left(\mathrm{kg} \mathrm{m}^{-2} \mathrm{~s}^{-1}\right)$, and $G_{i \rightarrow j}$ is the rate of phase change from the phase $i$ (source) to the phase $j$ (sink; $\mathrm{kg} \mathrm{m}^{-3} \mathrm{~s}^{-1}$ ).

For the liquid phase flow, a different transport mechanism is considered for each pore systems in wood (i.e., for cell wall and lumen). The cell wall is assumed to be in liquid-saturated state and the flux of (bound) water molecules is expressed by the diffusion equation. According to the activation energy of molecules in sorption sites, the effects of both the $T$ and $u$ are considered (Skaar 1998):

$$
\mathbf{g}_{b w}=-\mathbf{D}_{b w}\left(u_{b w}, T\right)\left[\frac{E_{b w} u_{b w}}{\left(R_{v} T-u_{b w} \partial E_{b w} / \partial u_{b w}\right) T} \nabla T+\nabla u_{b w}\right]
$$

where subscript $b w$ refers to the cell wall bound water, $\mathbf{D}_{b w}$ is the adsorbed liquid diffusivity tensor of the medium, and $E_{b w}$ is the activation energy of adsorbed molecules. In contrast to the bound water in the cell walls, the capillary transport in the lumens is described by Darcy's law, indicating the pressure gradient as the driving force for convective transport:

$$
\mathbf{g}_{f w}=-\rho_{l} \mathbf{K}_{l}\left(u_{f w}, T\right) \nabla p_{l}=-\rho_{l} \mathbf{K}_{l}\left(u_{f w}, T\right) \nabla\left(p_{\mathrm{g}}-p_{c}\right)
$$

where subscript fw refers to the free (bulk) water and $\mathbf{K}_{l}$ is the liquid permeability of the medium. Note that the gravitational effects are neglected in this equation.

For the gaseous phase flow, the gaseous phase (i.e., the mixture of water vapor and dry air) occupies only the lumen porosity and its transport mechanism includes bulk movement due to the pressure gradient as well as the diffusion of each component in the mixture. The convective (bulk) transport is described by Darcy's law:

$$
\mathbf{g}_{g, \text { conv. }}=-\rho_{g} \mathbf{K}_{g}(u, T) \nabla p_{g}=-\left(\rho_{g}^{v}+\rho_{g}^{a}\right) \mathbf{K}_{g}(u, T) \nabla p_{g}
$$

where subscript conv. refers to the convective part of the flux, $\rho_{g}$ is the density of gas phase, $\rho_{g}^{v}$ is the density of water vapor, $\rho_{g}^{a}$ is the density of dry air, and $\mathbf{K}_{\mathrm{g}}$ is the gas permeability of the medium. The diffusive part of the flow is described by the Fick's law of diffusion:

$$
\mathbf{g}_{v, \text { diff. }}=-\rho_{g} \mathbf{D}_{g}(u, T) \nabla\left(\frac{\rho_{g}^{v}}{\rho_{g}}\right)=\rho_{g} \mathbf{D}_{g}(u, T) \nabla\left(\frac{\rho_{g}^{u}}{\rho_{g}}\right)=-\mathbf{g}_{u, \text { diff. }}
$$

where subscript diff. refers to the diffusive part of the flux and $\mathbf{D}_{g}$ is the gas diffusivity tensor of the medium. Therefore, the flux vector of the water vapor and dry air can be written as follows:

$$
\mathbf{g}_{v}=-\rho_{g}^{v} \mathbf{K}_{g} \nabla p_{g}-\rho_{g} \mathbf{D}_{g} \nabla\left(\frac{\rho_{g}^{v}}{\rho_{g}}\right)
$$

$$
\mathbf{g}_{a}=-\rho_{g}^{a} \mathbf{K}_{g} \nabla p_{g}+\rho_{g} \mathbf{D}_{g} \nabla\left(\frac{\rho_{g}^{v}}{\rho_{g}}\right)
$$

In terms of energy conservation, by neglecting the mechanical work and in the absence of any internal heat sources, assuming local thermal equilibrium, the energy balance reads

$$
\sum_{j} w_{j} \frac{\partial h_{j}}{\partial t}=-\sum_{j} \mathbf{g}_{j} \cdot \nabla h_{j}+\nabla \cdot \boldsymbol{q}
$$

where $h_{j}$ is the specific enthalpy of component $j, \boldsymbol{q}$ is the conductive heat flux vector that is described by Fourier law, with the $T$ gradient as the driving force, and $\boldsymbol{q}=-\lambda \nabla T$, in which $\lambda$ is the thermal conductivity tensor of the medium, as per Lewis and Schrefler (1998).

The material properties related to heat and mass transport are next defined. The dry densities of EW and LW are selected as 300 and $680 \mathrm{~kg} \mathrm{~m}^{-3}$. These values give an overall density of $376 \mathrm{~kg} \mathrm{~m}^{-3}$ for dry wood based on a volume fraction of 4:1 for EW and LW. The density of liquid water is dependent on both $T$ and pressure. For water vapor and dry air, the ideal gas state equation, including both $T$ and pressure dependence, is used. The density of the moist wood is based on the volume fraction of the mentioned densities, thus implicitly including both $T$ and moisture effects.

Moisture content $u$ is a function of capillary pressure, or $\mathrm{RH}$, and of $T$. This hygroscopic curve is determined for the $T$ range of $25-100^{\circ} \mathrm{C}$ from Weichert (1963) and by extrapolation to $250^{\circ} \mathrm{C}$, as shown in Figure 2a.

The vapor diffusion coefficient is a function of RH, and as such also of capillary pressure and $T$, and given as an orthotropic tensor for EW and LW (see Figure 2b and c), according to Zillig (2009). The intrinsic liquid permeability is taken constant with a value at $T=296 \mathrm{~K}$ (Zillig 2009). The relative liquid permeability is given as a function of the saturation degree to the power eight for the $\mathrm{L}$ direction and power three for the transverse directions (Truscott 2004). The relative gas permeability decreases proportionally with the liquid saturation with power five in the $\mathrm{L}$ direction and power three in transverse directions (Truscott 2004). The activation energy $E_{b w}$ of the adsorbed water is a function of $u$ in the hygroscopic range (Skaar 1998):

$$
E_{b w}=2.23 \times 10^{6}-3.95 \times 10^{6} u+1.62 \times 10^{7} u^{2}-3.72 \times 10^{7} u^{3}
$$

The effective thermal conductivity tensor is dependent on $u$ and $T$ as reported by Kühlmann (1962) and is extrapolated to the range of $T$ considered here as follows. The thermal conductivity of wood is considered to be the superposition of the thermal conductivity of dry wood, water, and moist air. The thermal conductivity of dry wood increases linearly with $T$, whereas the thermal conductivity for both water and air is given as a function of $T$ in thermodynamics tables. Therefore, the thermal conductivity of wet wood includes the effects of $T$ and $u$. Note that the thermal conductivity of dry wood in the $\mathrm{L}$ direction is $2-2.5$ times greater than in the transverse directions. Similarly, the heat capacity of wet wood is given as a superposition of those of dry wood, water, and moist air (Forest Products Laboratory 2010).

The moisture-mechanical parameters are presented in Figure 1 and Table 1. The linear-logarithmic reduction of the stiffness with increasing $T$ in the range of $0-200^{\circ} \mathrm{C}$ is accounted for by scaling the stiffness matrix with a function of $T, f(T)$. According to the Forest Products Laboratory (2010), the components of the thermal expansion tensor of wood can be described as $\alpha_{L}=4.1 \times 10^{-6}$, $\alpha_{R}=32.4 \times 10^{-9} \rho_{\mathrm{dry}}+9.9 \times 10^{-6}$, and $\alpha_{T}=32.4 \times 10^{-9} \rho_{\mathrm{dry}}+18.4 \times 10^{-6}$. Note that, 

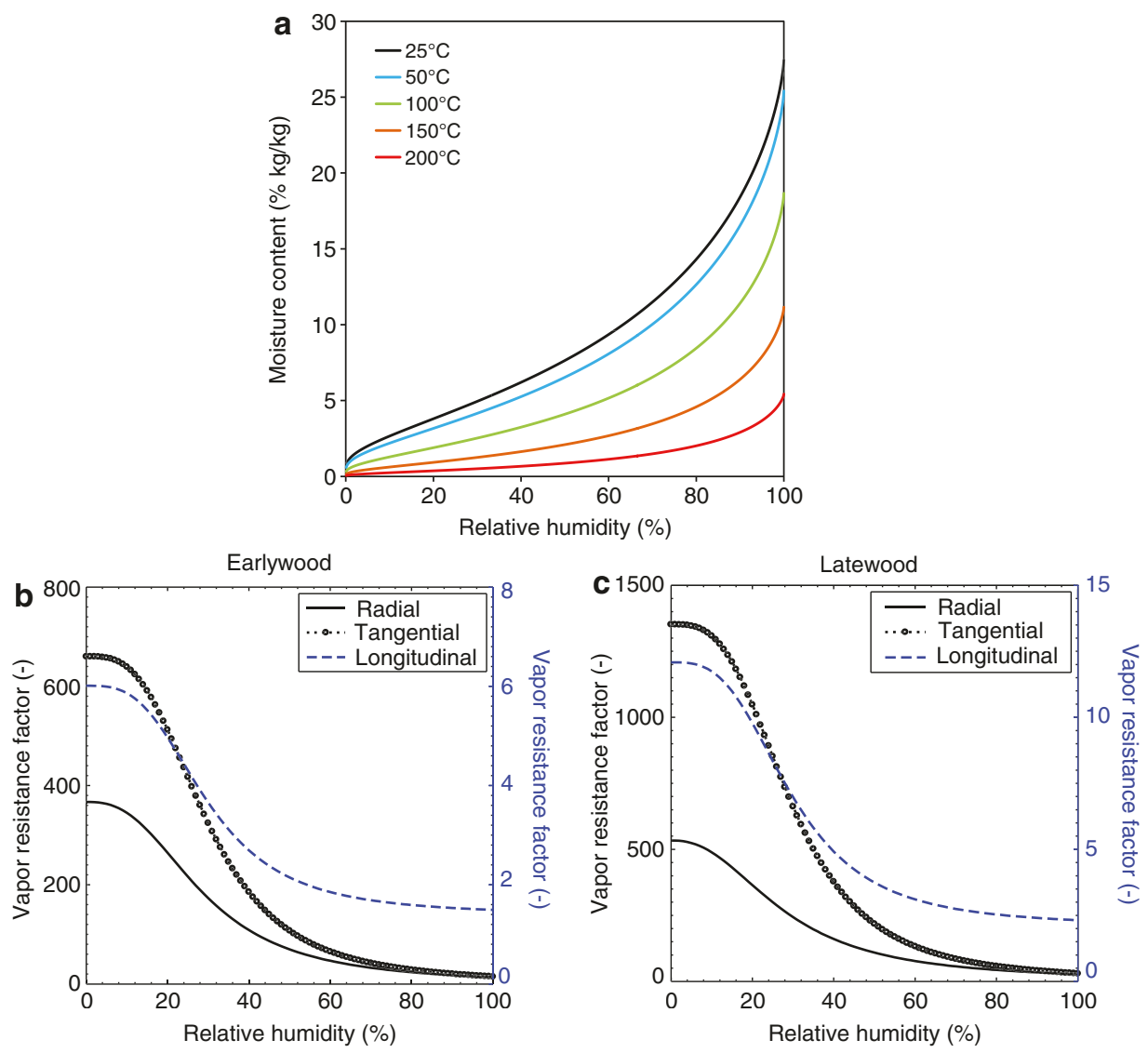

Figure 2: Mass transport material properties: (a) sorption isotherms of spruce measured at 25, 50, and 100 from Weichert (1963) and extrapolated at $150^{\circ} \mathrm{C}$ and $200^{\circ} \mathrm{C}$ and vapor resistance factor of (b) EW and (c) LW for the orthogonal directions as a function of RH.

due to a lack of data, all dependencies of the thermoporoelastic properties on $T$, effective pressure (or $u$ ), and mechanical stress cannot be considered.

Finally, the system of coupled nonlinear equations is solved by means of the FEM method with an in-house code, which follows an iterative total incremental approach (Moonen 2009) and which is able to solve fully coupled hygrothermo-mechanical problems.

\section{Validation experiment}

Samples of spruce, a softwood with an average density of $376 \mathrm{~kg} \mathrm{~m}^{-3}$ and dimensions $40 \mathrm{~mm}$ (height) $\times 80 \mathrm{~mm}$ (width) $\times 10 \mathrm{~mm}$ (thickness), are studied. The samples are sawn side-by-side out of the same wood planks, oriented so that the growth rings are visible during imaging and that heat flow along the height occurs perfectly in the L, R, or $\mathrm{T}$ direction. The samples are wrapped in Teflon tape to prevent mass loss and covered with two foam glass plates to minimize heat loss, at their vertical sides. The samples are oven dried to determine their mass and then conditioned until equilibrium in desiccators at $50 \%$ or $80 \% \mathrm{RH}$, corresponding to equilibrium moisture content of 31 and $53 \mathrm{~kg} \mathrm{~m}^{-3}$ (i.e., $8 \%$ and $14 \% u$ ).

The setup for the heating experiment is shown in Figure 3a. It consists of a frame that presses the sample firmly against a metallic heating foil of $50 \mu \mathrm{m}$ thickness with two long bolts. The setup and sample are at $25^{\circ} \mathrm{C}$ at the start of the experiment. Type $\mathrm{E}$ ( $\mathrm{NiCr}-\mathrm{CuNi})$ thermocouples are placed below the foil on top of an insulating ceramic base and inserted in the samples. A power supply of maximum power of $50 \mathrm{~A}$ and $120 \mathrm{~V}$ is applied for foil heating and the base thermocouples serve to feed back the $T$ to the power supply controller. The control system achieves a step increase to the desired foil $T$ in a few seconds followed by a perfectly maintained high $T$ of $150^{\circ} \mathrm{C}$ or $250^{\circ} \mathrm{C}$ over the duration of the test. During the test, the moisture and shrinkage behavior of the samples are documented with neutron radiography and the $T \mathrm{~s}$ detected with thermocouples are recorded by a data acquisition system.

The NI facility of the Neutron Transmission Radiography (NEUTRA) beamline at the Paul Scherrer Institute (PSI), Villigen, Switzerland, was used for imaging and 

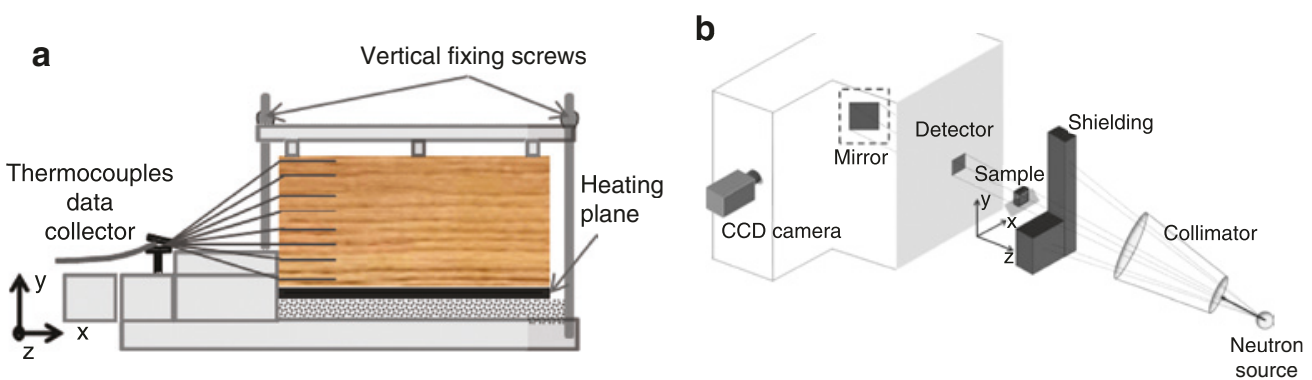

Figure 3: Schematic representations of (a) heating setup and (b) NI beamline.

quantification of time resolved $u$ in wood during the heating experiment. This instrument relies on a neutron beam within the thermal spectrum, with a most probable energy level of approximately $25 \mathrm{meV}$ (Lehmann et al. 2001). The schematic overview is presented in Figure $3 \mathrm{~b}$. The $\mathrm{x}$ - and $\mathrm{y}$-axes correspond to the detector plane axes, whereas the $\mathrm{z}$-axis shows the neutron beam direction. The detector consisted in a scintillator CCD camera system, with a total field of view of $87 \times 87 \mathrm{~mm}^{2}$. The scintillator is made of a $100-\mu \mathrm{m}$-thick sheet of zinc sulfide, doped with ${ }^{6} \mathrm{Li}$ as the neutron absorbing agent, to convert the neutron signals into visible light photons. The photons are then led via a mirror onto a cooled 16-bit CCD camera $(1024 \times 1024$ pixels). An initial neutron image is obtained once the sample is set within the frame as the reference image. Then, during the $20 \mathrm{~min}$ of the heating experiment, neutron images are acquired at regular intervals of $16 \mathrm{~s}$ with a spatial resolution of $100 \mu \mathrm{m}$.

The analysis of the neutron images for visualization of the $u$ distribution and their quantification is based on the intensity measurements of the transmitted neutron beam through an object. For a monochromatic beam, the transmitted intensity $I$ is described by the Lambert-Beer law:

$$
I=I_{0} e^{-\Sigma \cdot z}
$$

where $I_{0}$ is the intensity of the incident neutron beam, $z$ is the total thickness of the object along the neutron beam direction, and $\Sigma$ is the effective attenuation coefficient. Simplifying the composition of the samples consisting of wood and water, a bilayer approximation is used where the effect of moist wood on neutron attenuation is considered equivalent to the effect of a water layer with thickness $z_{w}$ added to the dry wood sample (Sedighi Gilani et al. 2012). Implementing this description, Equation (25) becomes

$$
I(t)=I_{0} e^{-\left\{\Sigma_{s} \cdot z_{s}+\Sigma_{w} \cdot z_{w}\right\}}
$$

where subscript $s$ refers to wood (solid) and $w$ refers to the equivalent water layer. At each time $t$ during the experiment, the change in the beam intensity with respect to the initial stage results from a $u$ change (i.e., the change in "effective" water layer thickness in the bilinear model). The $u$ change from the initial state (water mass per volume) is obtained by multiplying the change of effective water layer thickness $\Delta z_{w}(t)$ by the water density $\rho_{w}$ and dividing by the total sample thickness $z_{s}$. Finally, the obtained differential $u$ in water mass per volume is divided by the wood density to be presented in mass $\%(u)$.

A standard procedure, common to all radiation transmission-based imaging methods, is used to correct raw neutron images for artifacts. It includes correction for background noise of the CCD camera, for spatial fluctuations of the incident beam, for scattering by the experimental configuration and environment, and for polychromaticity of beam energy spectrum. The Quantitative Neutron Imaging algorithm developed at PSI (Hassanein 2006) is applied.

The desorption of water occurring during these heating experiments results in the shrinkage of the wood sample. $u$ is obtained from the logarithmic subtraction of the image taken at current time $t$ from the initial image taken at time $t=0$. However, as the sample changes geometry, the subtraction needs to be preceded by a registration of the image. Registration is achieved with a bilinear image registration algorithm, that is, TurboReg plug-in of ImageJ (Thevenaz et al. 1998), to scale back the image and align its edges with the edges of the reference image, at initial time.

\section{Model validation}

\section{Boundary conditions and numerical domain}

The calculation domain covers half of the specimens. In addition, to reduce the simulation time, a 2D approach is selected, assuming that the transport in the third direction is negligible. The wood sample is divided into several growth rings. For simplicity, all growth rings are assumed to be of the same $3 \mathrm{~mm}$ width, where each ring has an average density of $376 \mathrm{~kg} \mathrm{~m}^{-3}$, with $2.4 \mathrm{~mm}$ of EW at $300 \mathrm{~kg} \mathrm{~m}^{-3}$ and $0.6 \mathrm{~mm}$ of LW at $680 \mathrm{~kg} \mathrm{~m}^{-3}$ (Figure 4). 


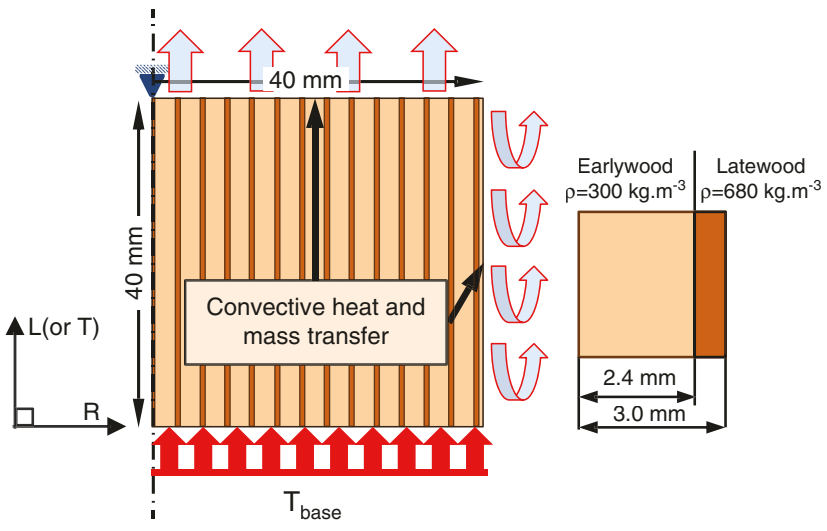

Figure 4: Computational domain and corresponding boundary conditions.

As seen in Figure 4, a constant temperature $T_{\text {base }}$ is applied at the bottom. For the symmetry plane, adiabatic boundary conditions for heat and no mass transfer are considered. For the right boundary, which is in contact with the environment, convective heat and mass transfer occurs. Thus, the boundary conditions with this regard are

$$
\begin{gathered}
\boldsymbol{q}_{T} \cdot \mathbf{n}=h_{\mathrm{T}}\left(T-T_{e}\right)+h_{M}\left(p_{v}-p_{v, e}\right)\left(c_{p, v}\left(T-T_{e}\right)+L_{v}\right) \\
+h_{M} c_{p, a}\left(p_{a}-p_{a, e}\right)\left(T-T_{e}\right) \\
\boldsymbol{q}_{M} \cdot \mathbf{n}=h_{M}\left(p_{v}-p_{v, e}\right) \\
p_{g}=\text { const. }
\end{gathered}
$$

where $\boldsymbol{q}_{T}$ and $\boldsymbol{q}_{M}$ are the heat and moisture flux vectors, respectively; $\mathbf{n}$ is normal vector of the surface; $T_{e}$ is the environment $T$; and $h_{T}$ and $h_{M}$ are the heat and moisture convective transfer coefficients, respectively. The mechanical boundary conditions include no displacement of the symmetry plane in the $\mathrm{R}$ direction as well as fixing at the top-left node; thus, the three surfaces are stress free.

\section{Validation: $u$ distribution versus time}

The samples at equilibrium with $80 \%$ and $50 \% \mathrm{RH}$ are considered after 5,10 , and $20 \mathrm{~min}$ of exposure to base $T \mathrm{~s}$ of $150^{\circ} \mathrm{C}$ and $250^{\circ} \mathrm{C}$, in terms of $u$ distribution in the RL plane, comparing experimental and simulation results in Figure 5 . The heat front is clearly imaged with the sharp $u$
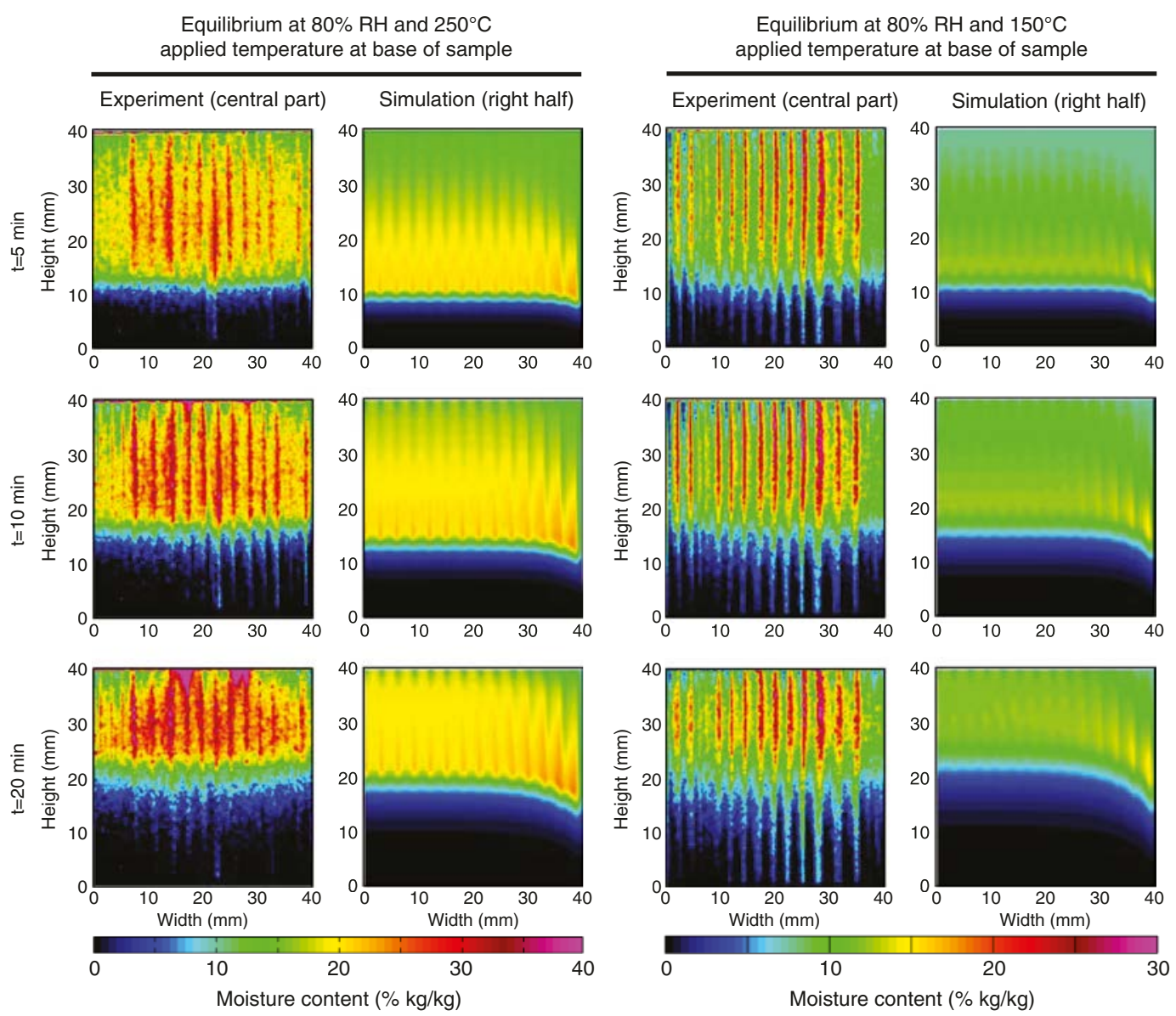

Figure 5: Moisture content distribution in the samples at different times, experimental results versus simulation results, in the R-L plane. 
front that is propagating along the height of the samples, at similar rate in both experimental and modeling results. In the experimental results, the growth ring pattern is more highlighted by the different $u$ levels, and the $u$ increase ahead of the heat front is more important than in the simulation results. These differences can be attributed to the higher inhomogeneity of the real material and some errors in the image processing. The difference between the $u$ s of EW and LW ahead of the heat front in the sample at 50\% $\mathrm{RH}$ is much less noticeable, indicating that the transport properties considered, although adequate for the sample at $80 \% \mathrm{RH}$, may have been different for this sample.

\section{Validation: $T$ and moisture content profiles versus time}

In this section, the average $T$ and $u$ across the samples are compared; thus, the profiles of $T$ and $u$ versus time at nine heights in the sample in Figure 6 are presented comparing
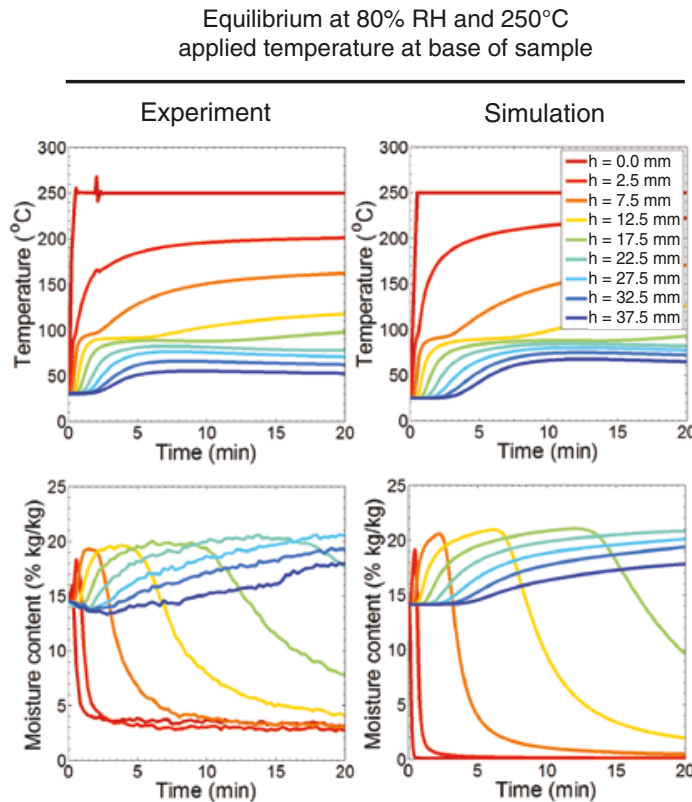

Equilibrium at $80 \% \mathrm{RH}$ and $150^{\circ} \mathrm{C}$ applied temperature at base of sample
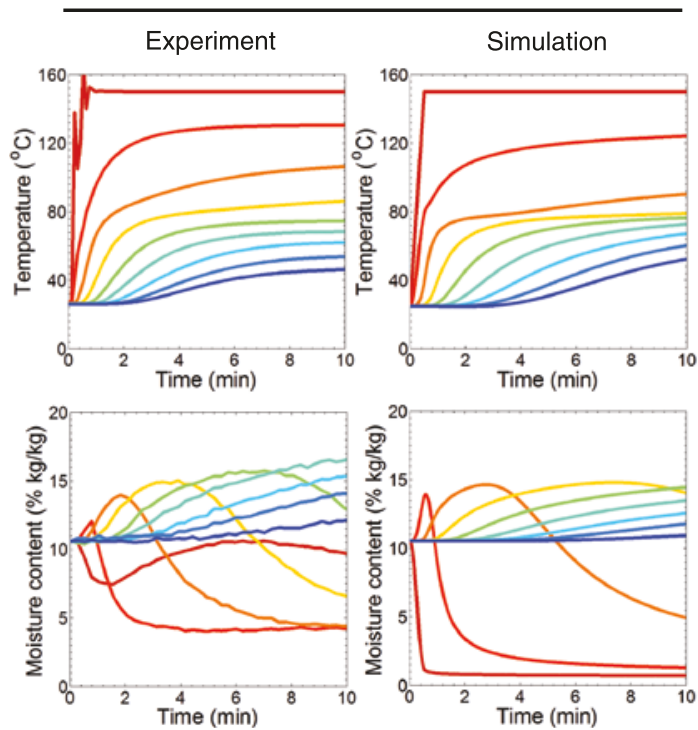

Equilibrium at $50 \% \mathrm{RH}$ and $250^{\circ} \mathrm{C}$ applied temperature at base of sample
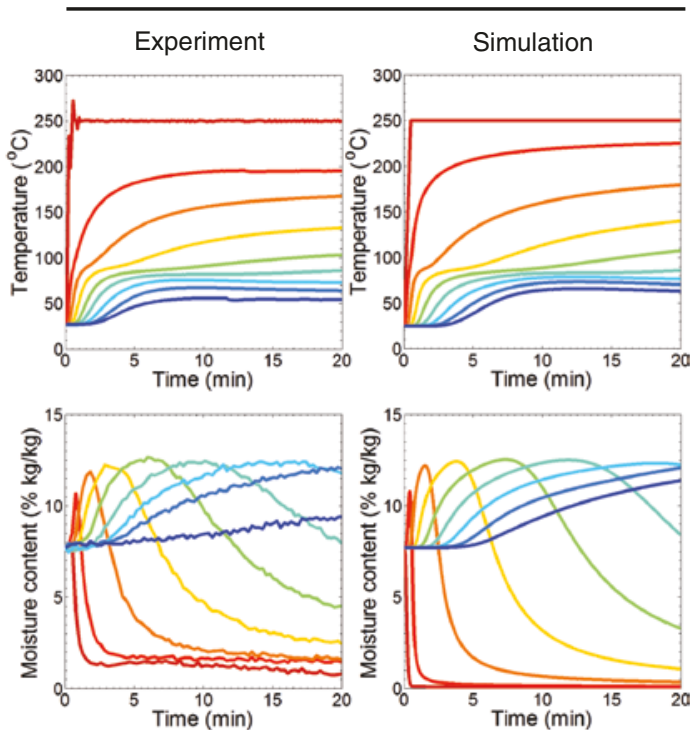

Equilibrium at $50 \% \mathrm{RH}$ and $150^{\circ} \mathrm{C}$ applied temperature at base of sample

Experiment

Simulation

$\mathrm{N} / \mathrm{A}$

Figure 6: Temperature and moisture content profiles versus time at nine heights in the samples, experimental results versus simulation results, in the R-L plane.

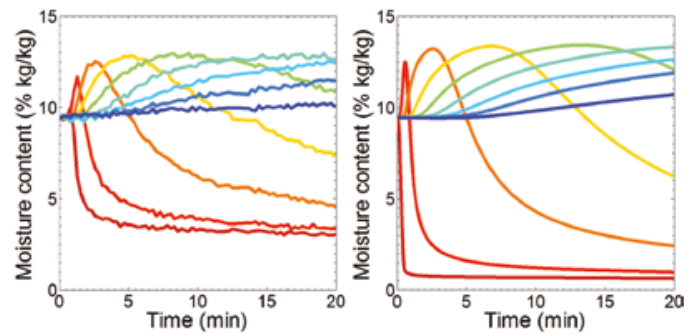


experimental and simulation results for four different sets of conditions. The $T$ profiles come from the thermocouples inserted in the sample. The $u$ profiles are taken from the calibrated neutron images presented in part in Figure 5. $u$ is averaged in narrow bands ( $0.50 \mathrm{~mm}$ thickness) across the growth ring in the same height as the thermocouples. Figure 6 shows very clearly that the global heat and moisture behavior of the four samples as documented experimentally is well captured by the simulations. In terms of $T$, the rate of increase and the final values after $20 \mathrm{~min}$ are correctly captured. The missing data of the sample at equilibrium with $50 \% \mathrm{RH}$ and exposed to a $T$ of $150^{\circ} \mathrm{C}$ are due to a computer malfunction during the experiment. Regarding $u$, the increase of $u$ ahead of the heat front is very well captured as well as the length of the plateaus and the rate of drying. However, the model does not capture the residual $u$ after drying.

\section{Validation: surface T profiles versus time}

To capture the $T$ field of the sample during the heating process, IRT is carried out by means of an NEC TH 3102 camera system with a Stirling cooler for dry sample and moist sample (exposed to $80 \% \mathrm{RH}$ ), without insulation to allow IR measurements. The $T$ fields of four samples from IRT and the corresponding plots from the modeling are presented in Figure 7 at $t=10 \mathrm{~min}$. Some differences between the results from modeling and the experimental measurements are visible. In this experiment, in contrast to the NEUTRA experiment, the (larger) sides of the samples are in contact with the environment, so there is a considerable amount of convective heat loss from these sides (i.e., heat transfer, which is not included in the 2D model). This effect is more considerable for the dry samples because of lower heat capacity and thermal conductivity of the medium. In addition, it should be noted that the experiment results show the surface $T$, whereas the model contours actually show the middle $T$ distribution of the sample.

In this experiment, several thermocouples are also embedded in the middle plane of the sample and at different heights. The recorded values by thermocouples and averaged values at similar height from simulation are presented in Figure 8. From these figures, the role of water on the formation of $T$ plateaus is clearly illustrated, as no plateau is seen in the $T$ profiles of the dry samples.

\section{Validation: shrinkage profiles versus time}

Finally, to check the validity of the poromechanics approach, the shrinkage profiles of the sample with $80 \%$ $\mathrm{RH}$ and base $T$ of $250^{\circ} \mathrm{C}$ are determined from the neutron
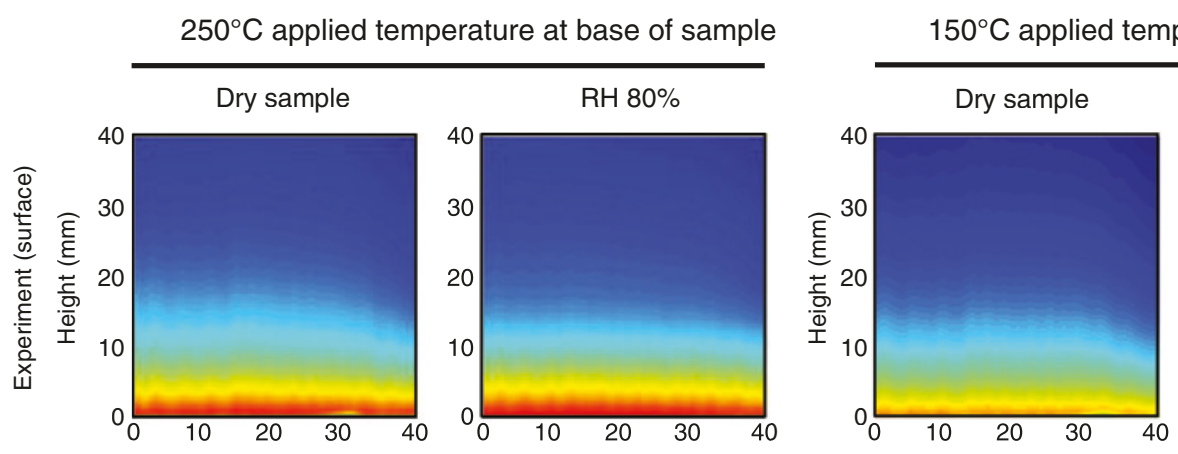

$150^{\circ} \mathrm{C}$ applied temperature at base of sample
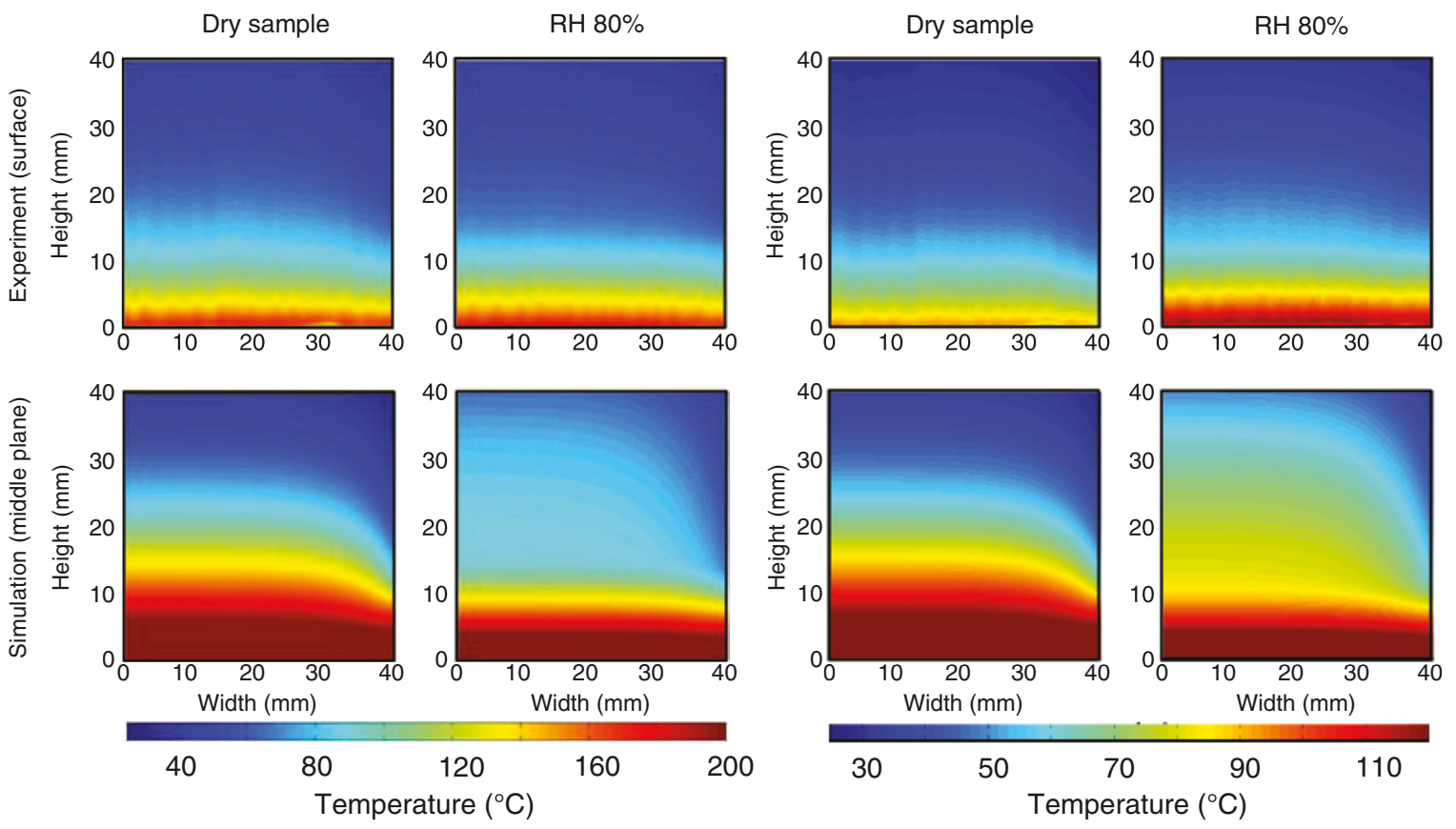

Figure 7: Temperature distribution in the samples at different times, experimental results versus simulation results, in the R-L plane. 

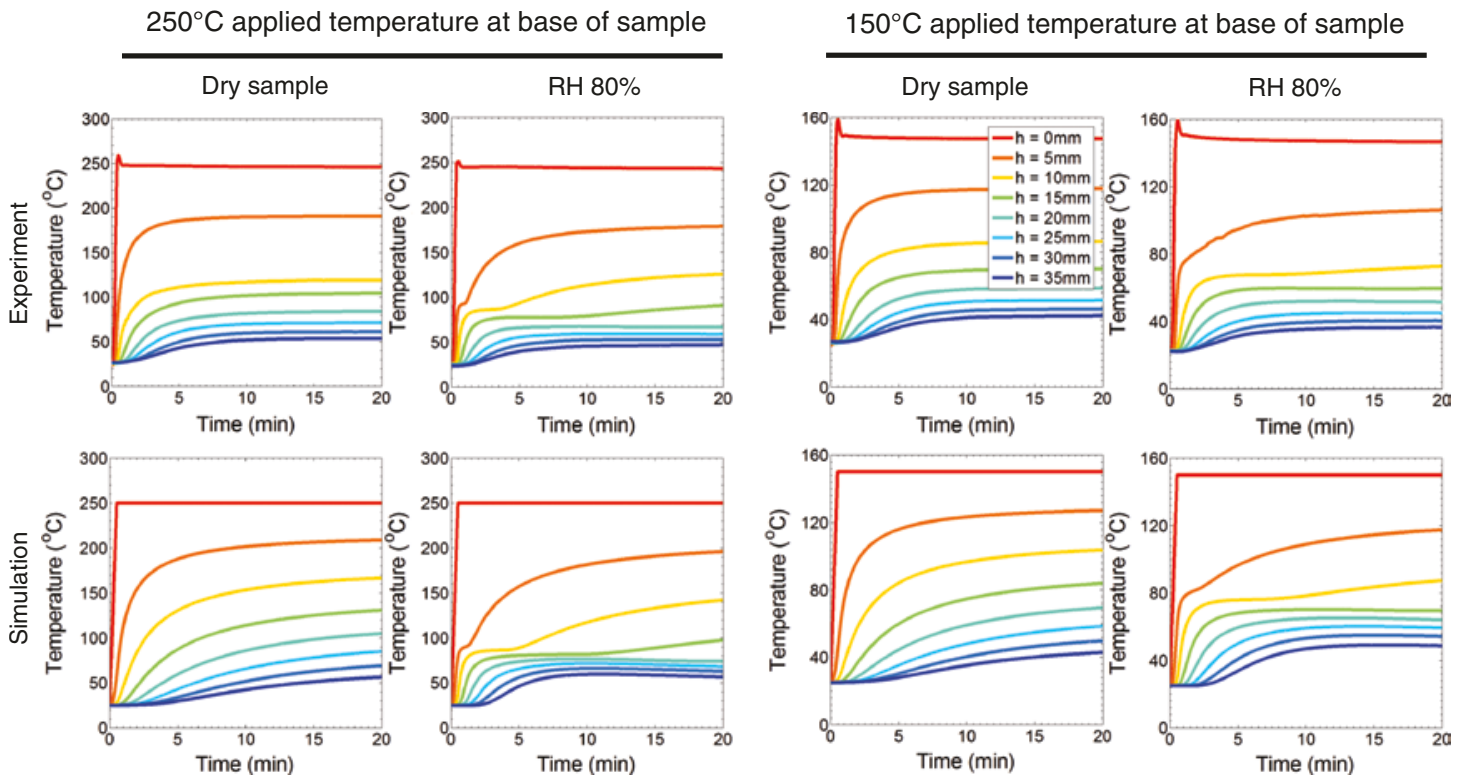

Figure 8: Temperature profiles versus time at nine heights in the samples, experimental results versus simulation results, in the R-L plane.

images and for different time steps evaluated by image digitizing software. By considering these deformations of the sample edge, it is possible to find the average strain profiles of the sample along its height as (Sedighi Gilani et al. 2014)

$$
\varepsilon_{R}(y, t)=\frac{L_{R}(y, t)-L_{R}\left(y, t_{0}\right)}{L_{R}\left(y, t_{0}\right)}
$$

where $\varepsilon_{R}(y, t)$ is the time-dependent average strain in the $\mathrm{R}$ direction and $L_{R}(y, t)$ corresponds to the dimension of the sample at time $t$ along the R axis at a height position $y$. For the model, the same equation is used to find the average strain. The results are presented in Figure 9. As demonstrated, both tensile and compressive strains occur along the height of the sample, which is indicative of a bending behavior. The moment causing this bending comes from the induced internal stress due to the moisture loss, thus related to pore pressure, at the region near the heat source and the gain of moisture ahead of the heating front. In all appearances, the negative strain zone is due to the hygric effects and thus is considered to be shrinkage. However, the positive strains could be due to the mechanical effects of balancing the moment, not only at higher moisture content, and thus may not be purely swelling strains.
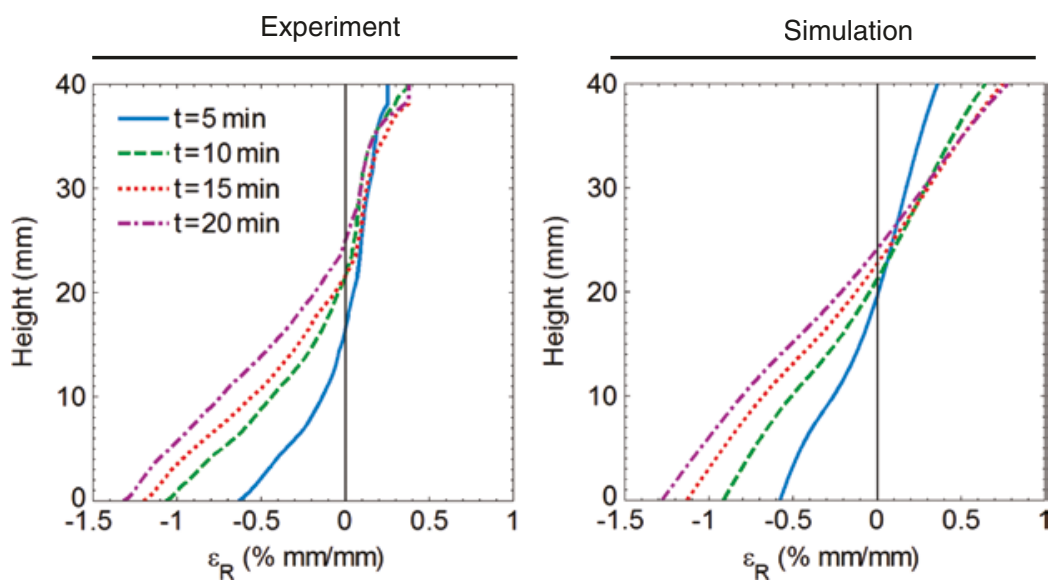

Figure 9: Average strain profiles in the R direction along the height of the sample at different time steps and for the sample with equilibrium with $80 \% \mathrm{RH}$ and $T_{\text {base }}=250^{\circ} \mathrm{C}$. 


\section{Conclusions}

The exact prediction of the thermal, hygric, and mechanical behavior of wood requires a holistic consideration of several parameters. A fully coupled poromechanical approach is proposed in the present paper and the predictions are validated with NI measurements of moist wood specimens exposed to high temperature. It was demonstrated that the model captures accurately the variations of moisture content, temperature, and dimensions in the course of one-side heating. The model allows for the implementation of different boundary conditions. The implementation of the mesoscale features of the growth rings (EW and LW) is necessary for exact predictions.

Acknowledgments: The contributions of Stefan Carl and Roger Vonbank in developing the experimental setups are acknowledged. Neutron radiography was performed at Neutra beamline, SINQ, PSI, Villigen, Switzerland. SNF Sinergia grant no. 127467 and the COST Action FP0904 of the EU RTD Framework Programme are acknowledged.

\section{References}

Abbasion, S., Moonen, P., Carmeliet, J., Derome, D. (2015) A hygrothermo-mechanical model for wood: part B. Parametric studies and application to wood welding - COST Action FP0904 2010-2014: Thermo-Hydro-Mechanical wood behavior and processing. Holzforschung 69:839-849.

Biot, M.A. (1941) General theory of three-dimensional consolidation. J. Appl. Phys. 12:155-164.

Carmeliet, J., Derome, D., Dressler, M., Guyer, R. (2013) Nonlinear poro-elastic model for unsaturated porous solids. J. Appl. Mech. 80:020909.

Coussy, O. (1989) Thermodynamics of saturated porous solids in finite deformation. Eur. J. Mech. A Solids 8:1-14.

Coussy, O. Mécanique des Milieux Poreux. Technip, Paris, 1991. In English, Mechanics of Porous Continua. Wiley, Chichester, 1995.

Coussy, O. Poromechanics. Wiley, Chichester, 2004.

Coussy, 0. (2007) Revisiting the constitutive equations of unsaturated porous solids using a Lagrangian saturation concept. Int. J. Numer. Anal. Meth. Geomech. 31:1631-1713.

Coussy, O. Mechanics and Physics of Porous Solids. John Wiley \& Sons, UK, 2010.

Coussy, O., Eymard, R., Lassabatère T. (1998) Constitutive modelling of unsaturated drying deformable materials. J. Eng. Mech. ASCE 124:658-667.

D’Alvise, L., Massoni, E., Walloe, S.J. (2002) Finite element modeling of the inertia friction welding process between dissimilar materials. J. Mat. Proc. Technol. 125-126:387-391.

Derome, D., Griffa, M., Koebel, M., Carmeliet, J. (2011) Hysteretic swelling of wood at cellular scale probed by phase contrast X-ray tomography. J. Struct. Biol. 173:180-190.
Ganne-Chédeville, C., Duchanois, G., Pizzi, A., Leban, J.-M., Pichelin, F. (2008) Predicting the thermal behaviour of wood during linear welding using the finite element method. J. Adhesion Sci. Technol. 22:1209-1221.

Gawin, D., Pesavento, F., Schrefler, B.A. (2003) Modelling of hygro-thermal behaviour of concrete at high temperature with thermo-chemical and mechanical material degradation. Comput. Methods Appl. Mech. Eng. 192:1731-1771.

Forest Products Laboratory (2010) Wood Handbook - Wood as an engineering material. General Technical Report. FPL-GTR-190. Madison, WI: U.S. Department of Agriculture, Forest Service, Forest Products Laboratory: $508 \mathrm{p}$.

Fu, L., Duan, L. (1998) The coupled deformation and heat flow analysis by finite element method during friction welding. Welding J. 77:202-207.

Hassanein, R. (2006) Correction methods for the quantitative evaluation of thermal neutron tomography. Ph.D. dissertation, ETHZ Zurich, Switzerland.

Lehmann, E., Vontobel, P., Wiezel, P. (2001) Properties of the radiography facility NEUTRA at SINQ and its potential for used as European reference facility. Nondestr. Test. Eval. 16:191-202.

Lewis, R.W., Schrefler, B.A. The Finite Element Method in the Static and Dynamic Deformation and Consolidation of Porous Media. Second Edition. John Wiley \& Sons Ltd., 1998, Reprint, 2000.

Lindemann, Z., Skalski, K., Wlosinski, W., Zimmerman, J. (2006) Thermo-mechanical phenomena in the process of friction welding of corundum ceramics and aluminium. B Polish Acad. Sci. 54:1-8.

Kühlmann, G. (1962) Investigation of the thermal properties of wood and particleboard in dependency on moisture content and temperature in the hygroscopic range (GER). Holz Roh- Werks. 20:259-70.

Maloney, T.C., Paulapuro, H. (1999) The formation of pores in the cell wall. J. Pulp Pap. Sci. 25:430-436.

Moonen, P. (2009) Continuous-discontinuous modelling of hygrothermal damage processes in porous media. Ph.D. thesis, Katholieke Universiteit Leuven.

Neuhaus, F.H. (1981) Elastizitätszahlen von Fichtenholz in Abhängigkeit von der Holzfeuchtigkeit. Ph.D. thesis, Institut für konstruktiven Ingenieurbau Ruhr-Universität Bochum.

Patera, A., Derome, D., Griffa, M., Carmeliet, J. (2013) Hysteresis in swelling and in sorption of wood tissue. J. Struct. Biol. 182:226-234.

Rémond, R., Passard, J., Perré, P. (2007) The effect of temperature and moisture content on the mechanical behaviour of wood: a comprehensive model applied to drying and bending. Eur. J. Mech. A Solids 26:558-572.

Sedighi Gilani, M., Griffa, M., Mannes, D., Lehmann, E., Carmeliet, J., Derome, D. (2012) Visualization and quantification of liquid water transport in softwood by means of neutron radiography. Int. J. Heat Mass Transfer 55:6211-6221.

Sedighi Gilani, M., Abbasion, S., Lehmann, E., Carmeliet, J., Derome, D. (2014) Neutron imaging of moisture displacement due to steep temperature gradients in hardwood. Int. J. Thermal Sci. 81:1-12.

Skaar, C. Wood-Water Relations. Springer-Verlag, Berlin, Germany, 1998.

Sluzalec, A. (1990) Thermal effects in friction welding. Int. J. Mech. Sci. 32:467-478. 
Thevenaz, P., Ruttimann, U.E., Unser, M. (1998) A pyramid approach to subpixel registration based on intensity. IEEE Trans. Image Process. 7:27-41.

Truscott, S. (2004) A heterogeneous three-dimensional computational model for wood drying. Ph.D. thesis, School of Mathematical Sciences, Queensland University of Technology.

Weichert, L. (1963) Investigations on sorption and swelling of spruce, beech and compressed-beech wood at temperatures between 20 and $100^{\circ} \mathrm{C}$. Holz Roh- Werks. 21:290-300.
Zeng, Q., Fen-Chong, T., Dangla, P., Li, K. (2011) A study of freezing behavior of cementitious materials by poromechanical approach. Int. J. Solids Struct. 48:3267-3273.

Zillig, W. (2009) Moisture transport in wood using a multiscale approach. Ph.D. thesis (Eng.), Building Physics Laboratory, Katholieke Universiteit Leuven.

Zoulalian, A., Pizzi, A. (2007) Wood dowel rotation welding a heat transfer model. J. Adhesion Sci. Technol. 21: 97-108. 